\title{
A rare case of retroperitoneal paraganglioma located in the neck of the pancreas: a case report and literature review
}

\author{
Wenchao Wang ${ }^{1}$, Yunsheng Qin ${ }^{1}$, Huifang Zhang ${ }^{2}$, Kangjie Chen ${ }^{1}$, Zhengtao Liu ${ }^{1}$, Shusen Zheng ${ }^{1}$ \\ ${ }^{1}$ Division of Hepatobiliary and Pancreatic Surgery, Department of Surgery, First Affiliated Hospital, School of Medicine, Zhejiang University, \\ Hangzhou, China; ${ }^{2}$ Department of Pathology, First Affiliated Hospital, School of Medicine, Zhejiang University, Hangzhou, China \\ Correspondence to: Shusen Zheng, PhD. Division of Hepatobiliary and Pancreatic Surgery, Department of Surgery, First Affiliated Hospital, School of \\ Medicine, Zhejiang University, No. 79 Qingchun Road, Hangzhou 310003, China. Email: shusenzheng@zju.edu.cn.
}

\begin{abstract}
Paraganglioma (PGL) is an uncommon tumor located in the head, neck and abdomen. The majority of the tumor is benign and the patient has no obvious clinical symptoms. However, PGL located in the pancreas is rather rare and tends to mimic Castleman's disease, pancreatic neuroendocrine tumors and pancreatic primary tumor. Herein, we reported a patient with PGL that occurred in the neck of the pancreas. A 75-year-old Chinese female presented to our hospital with a complaint of upper abdomen pain for two weeks and she had good past health. The laboratory findings and physical examination were all normal. Preoperative computed tomography (CT) and magnetic resonance imaging revealed a tumor located in the neck of the pancreas and a tentative diagnosis of Castleman's disease or PGL was made. We resected the tumor by laparoscopic surgery. Postoperative pathology and immunohistochemistry confirmed that the tumor was a PGL. The patient was recovered well after a postoperative follow-up of 6 months. PGL located in the neck of the pancreas is difficult to be diagnosed accurately and clinicians have difficulties in distinguishing PGL from Castleman's disease, pancreatic neuroendocrine tumors and pancreatic primary tumor. Fifteen cases were listed to show the characters of PGL located in the pancreas and we also presented the difference among PGL, Castleman's disease and pancreatic neuroendocrine tumor. We showed our experience of treating such a rare tumor hoping to help clinicians correctly diagnose and treat PGL.
\end{abstract}

Keywords! Laparoscopic technique; paraganglioma (PGL); pancreas; Castleman's disease

Submitted Oct 13, 2020. Accepted for publication Feb 23, 2021.

doi: $10.21037 /$ gs-20-758

View this article at: http://dx.doi.org/10.21037/gs-20-758

\section{Introduction}

Paraganglioma (PGL) is a rare vascular, neuroendocrine tumor of the paraganglia cell cluster originated from the neural crest that has co-migration with the autonomic nervous system (1). Majority of the tumor are benign as only $10 \%$ of them are malignant and patients often have no obvious clinical symptoms. The morbidity of PGL approximately lies between 1:6,500 and 1:2,500, with a prevalence of 500 to 1,600 patients every year in America (2). About 3\% of PGLs happen in the head and neck (3). The common location is retroperitoneal accounting for up to $77 \%$ and the PGL is often described as a mass around the corpora paraaortic (4). However, PGL located in the pancreas is rather rare and tends to mimic Castleman's disease or pancreatic neuroendocrine tumor(pNET). Herein, we reported a patient with PGL that occurred in the pancreatic neck. We presented the following case in accordance with the CARE reporting checklist (available at http://dx.doi. org/10.21037/gs-20-758).

\section{Case presentation}

A 75-year-old Chinese female was admitted to our hospital with a complaint of upper abdomen discomfort for two weeks and she had good past health. The family and psychosocial history were not special. The patient had no family history of PGL. She had no relevant past interventions. 


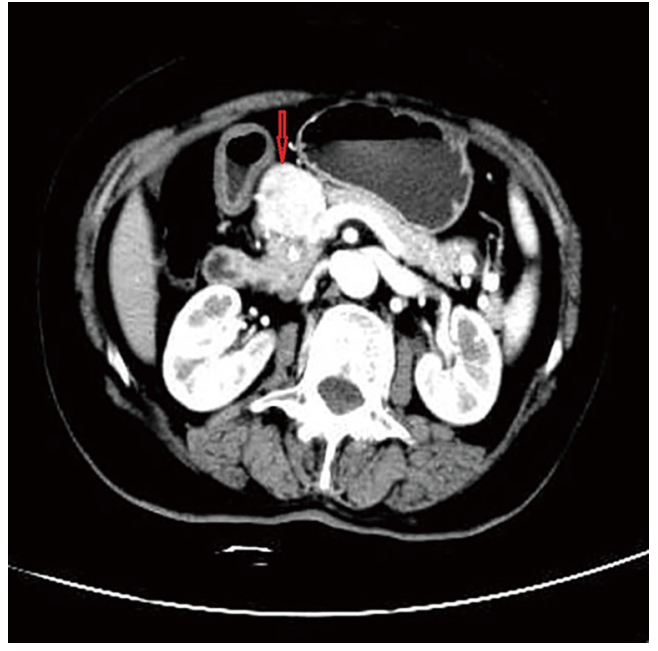

Figure 1 Computed tomography-enhanced scan shows a $3.1 \mathrm{~cm} \times 3.8 \mathrm{~cm}$ mass with abundant blood in the neck of the pancreas, significantly enhanced during the arterial phase and have a smooth and well-defined boundary. There aren't significant enlarged retroperitoneal lymph nodes. No obvious abnormalities are observed in the liver, gallbladder, spleen, kidney and gastrointestinal tract. The mass could be Castleman's disease.

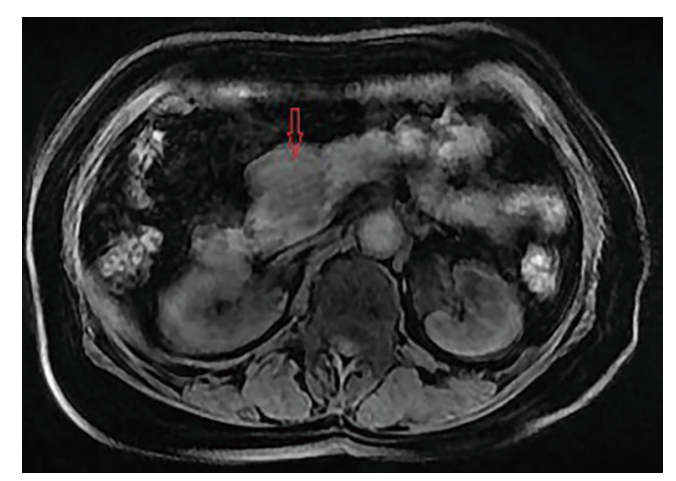

Figure 2 Magnetic resonance imaging (MRI) reveals an abdominal mass in the pancreatic neck. The mass has clear outline, with a fine boundary to the pancreas. It is obviously enhanced after enhanced scan, and vascular shadow are visible at the edge. It could be Castleman's disease and please exclude paraganglioma based on clinical practice.

The laboratory findings and physical examination were normal. Especially, the tumor marker for alpha-fetoprotein (AFP, $1.3 \mathrm{ng} / \mathrm{mL}$ ), carcinoembryonic antigen (CEA, $2.7 \mathrm{ng} / \mathrm{mL})$, carbohydrate antigen 199 (CA199, <2 U/mL), carbohydrate antigen 125 (CA125, $22.5 \mathrm{U} / \mathrm{mL})$, serum ferritin (40.1 ng/mL), carbohydrate antigen 153 (CA153,
$10.8 \mathrm{U} / \mathrm{mL}$ ) were all in the normal range. It was normal for the catecholamine in the blood (Norepinephrine: $1.0 \mathrm{nmol} / \mathrm{L}$, Epinephrine: $180 \mathrm{pmol} / \mathrm{L}$ ) and urine (Norepinephrine: $221.5 \mathrm{nmol} / 24 \mathrm{~h}$, Epinephrine: $183.6 \mathrm{nmol} / 24 \mathrm{~h}$ ) and the patient didn't have paroxysmal hypertension or tachycardia. An enhanced abdominal computed tomography scan (CT, Figure 1) showed a $3.1 \mathrm{~cm} \times 3.8 \mathrm{~cm}$ mass with abundant blood in the neck of the pancreas, significantly enhanced during the arterial phase and had a smooth and welldefined boundary. There were no significant enlarged retroperitoneal lymph nodes. No obvious abnormalities were observed in the liver, gallbladder, spleen, kidney and gastrointestinal tract. The mass could be Castleman's disease. Magnetic resonance imaging (MRI, Figure 2) revealed an abdominal mass in the pancreatic neck. It presented hypointense or isointense on T1WI and hyperintense on T2WI. The mass had a clear outline, with a fine boundary to the pancreas. It was obviously enhanced after the enhanced scan, and vascular shadow was visible at the edge. It could be Castleman's disease and please exclude PGL based on clinical practice. We suspected that this tumor was a PGL, Castleman's disease, or pNET.

The tumor's size $(3.1 \mathrm{~cm} \times 3.8 \mathrm{~cm})$ was large and the patient had obvious abdominal pain, which was indication of surgery. The puncture has certain risks and it can only obtain a small amount of cytopathological tissue, which isn't helpful for accurate diagnosis of tumor type. Therefore, we decided to perform laparoscopic exploratory surgery firstly, and send the tumor to perform intraoperative frozen section pathological diagnosis after resection. The operative way was determined by the intraoperative freezing pathological results.

The patient underwent laparoscopic surgery and in the process of the operation, we found the tumor was surrounded by vessels. We carefully separated the tumor from the pancreatic tissue, and eventually removed it completely. The tumor with a size of $3.1 \mathrm{~cm} \times 3.8 \mathrm{~cm} \mathrm{had}$ a complete envelope (Figure 3). Intraoperative frozensection examination: The tumor is rich in blood, waiting for the definite diagnosis of routine pathology and immunohistochemistry. Considering the high possibility of benign mass, we only performed simple tumor resection. Postoperative pathology (hematoxylin \& eosin staining): the tumor cells are polygonal, the chromatin of cells is fine, the cytoplasm of cells is abundant and tumor cells are arranged in clusters or nests (Figure 4). Immunohistochemistry: CK (pan) (-), Ki-67(+, <1\%,), Melan A (-), chromogranin $(\mathrm{Cg}$ A) (+), synaptophysin (Syn) (+), S-100 (+) (Figure 4). It 
was diagnosed as a PGL. The patient recovered well and examination findings were normal after a postoperative follow-up of 6 months. The patient's compliance was good and there were no adverse or unanticipated events. The timeline for the patient is outlined in Figure 5. All procedures performed in studies involving human participants were in accordance with the ethical standards of the institutional and/or national research committee(s) and with the Helsinki Declaration (as revised in 2013).

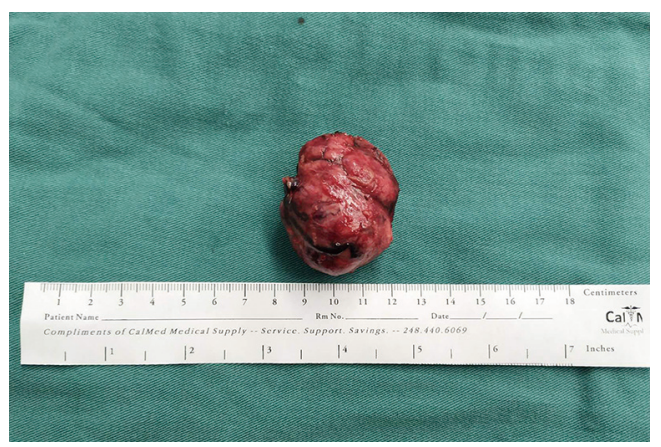

Figure 3 The tumor is completely removed and the size of the tumor is about $3.1 \mathrm{~cm} \times 3.8 \mathrm{~cm}$.
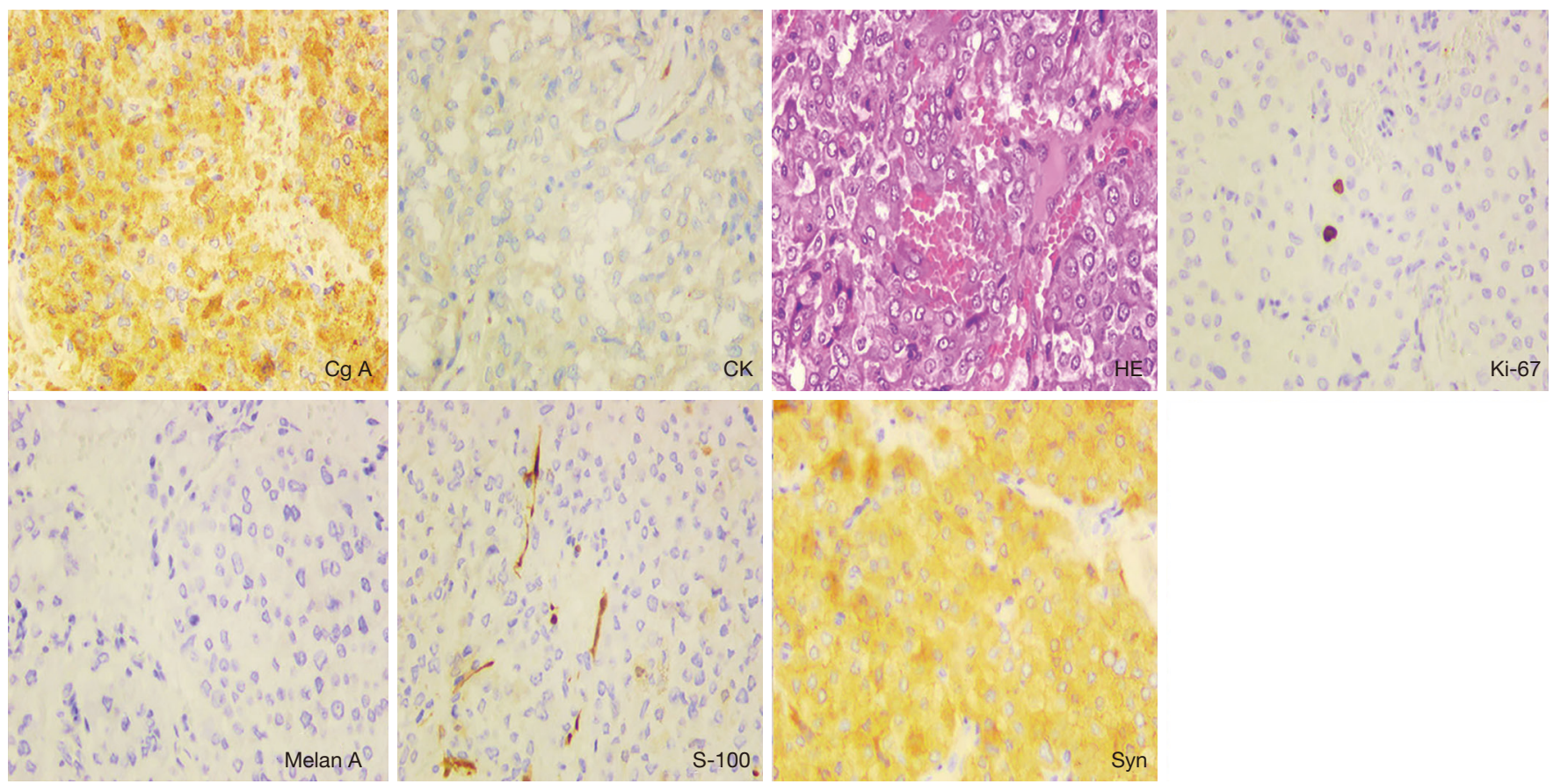

Figure 4 Pathology suggests that the tumor cells are polygonal, the chromatin is fine, the cytoplasm is rich, transparent or fine-grained, arranged in clusters or nests, and the blood vessels are rich (hematoxylin \& eosin staining, magnification 200x). Immunohistochemistry: CK (pan) (-), Ki-67 (+, <1\%,), Melan A (-), Cg A (+), Syn (+), S-100 (supporting cells+) (magnification 200×).

Written informed consent was obtained from the patient. The study was approved by the ethics committee of The First Affiliated Hospital, School of Medicine, Zhejiang University (No. IIT20200277A).

\section{Discussion}

PGL deriving from the thorax, abdomen and pelvic regions overproduces some hormones, especially norepinephrine, but PGL derived from the head and neck region is generally biochemically silent (5). PGL was mainly presented to be local compression symptoms and some of them produce catecholamine which causes hypertension. Therefore, surgeons need to measure the catecholamine of the patient in plasma and urine before surgery. In this case, the patient's blood pressure was normal. PGL which is originated from the periphery of the human aorta or the beginning of the inferior mesenteric artery is called retroperitoneal PGL and Color Doppler ultrasound, CT, MRI often show a round, elliptical, or lobulated, of varying sizes, and enveloped mass (6). In this case, a tumor located in the pancreas so differential diagnosis ought to be considered including pancreatic pseudocysts, intraductal papillary mucinous 


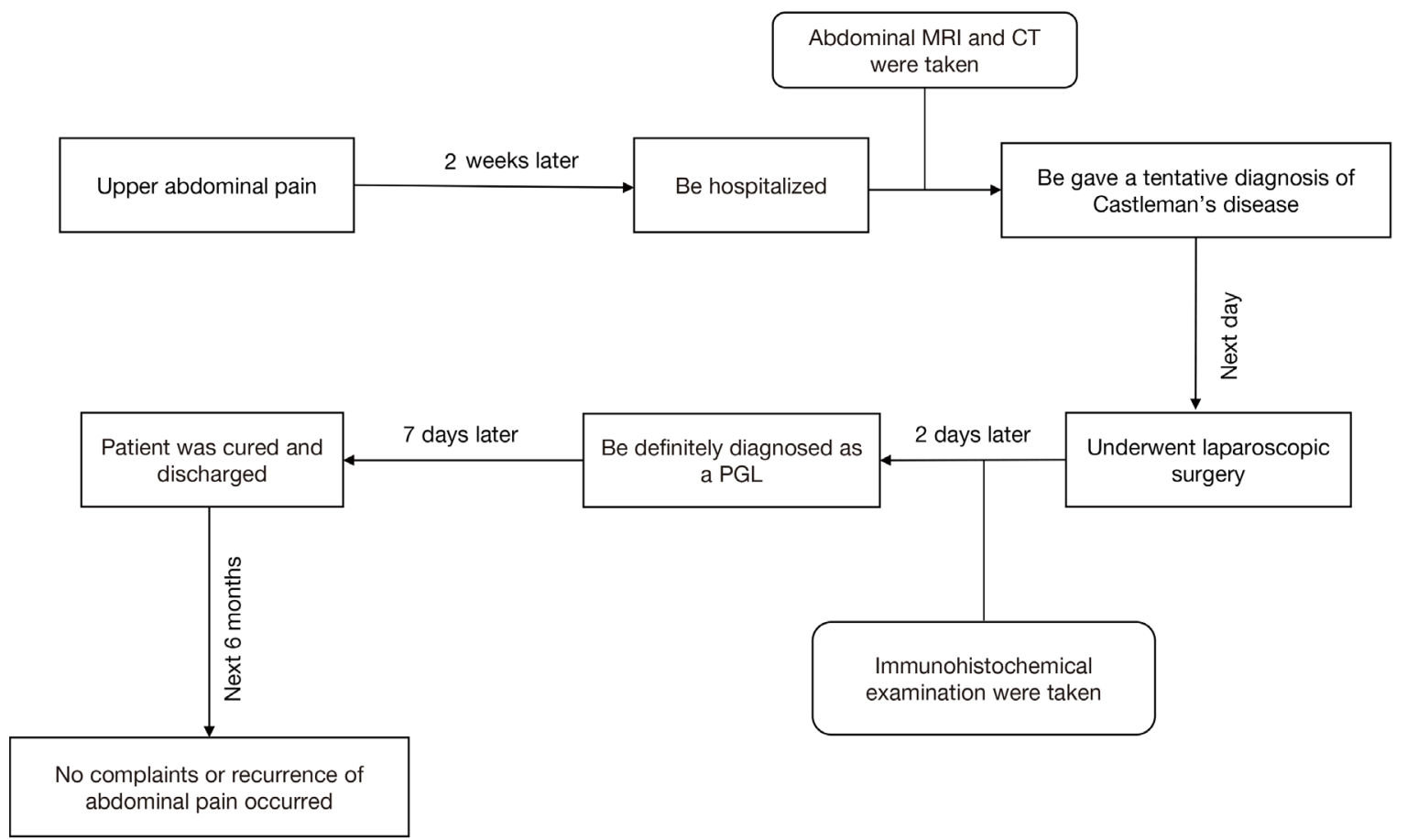

Figure 5 The timeline for the case.

neoplasm (IPMN), mucinous cystic neoplasm (MCN), serous cystic neoplasm (SCN), solid pseudopapillary neoplasm (SPN), pancreatic neuroendocrine tumor(pNET) and pancreatic adenocarcinoma. The surgeon should consider rare diseases for the differential diagnosis of uncommon strongly enhanced retroperitoneal masses. Nowadays, [68Ga] DOTA-SSA PET/Ct may be the preferred imaging modality of retroperitoneal PGL, especially for patients with SDHx mutations (7).

However, imaging examinations often lack specificity between PGL and Castleman's disease. Ultrasound-guided fine-needle aspiration (EUS-FNA) biopsy can provide a sufficiently accurate specimen, which is valuable for identifying tumors. Layfield et al. (8) reported the diagnostic accuracy of EUS-FNA for retroperitoneal tumors was $74.0 \%$. However, Immunohistochemistry can't be gained just by FNA so surgical resection remained necessary for a definitive diagnosis. With the maturity of laparoscopic techniques, it is possible to treat retroperitoneal tumors by laparoscopic surgery. Walz et al. (9) had reported 9 cases of retroperitoneal PGL, which were removed by laparoscopic surgery with a lower incidence of complications. In this case, laparoscopic resection for the tumor was performed, and the operation was successful with good postoperative recovery. Most of the retroperitoneal tumors are a local recurrence, and there is still the possibility of re-excision. Some studies have found that the resection of adjacent organs with tumors $>7.0 \mathrm{~cm}$ in diameter may have important implications for prognosis and of course, need to be fully evaluated before surgery (10). Aggressive surgery should be performed as long as the patient's general condition is well, with no distant or extensive abdominal metastases.

We choose cases diagnosed with PGL located in the pancreas from 14 articles through PubMed from 2001 to 2019 (Table 1) (11-24). These patients' age ranged from 30 to 72 years old and only $3 / 15$ were males. Eight patients $(11,15,16,18,20-23)$ were presented to be abdominal discomfort and six $(12,14,17,19,23,24)$ cases were asymptomatic. Only one special patient (13) presented with loss of appetite, weight loss, weakness, and breathlessness. CT was performed in 14 case $(11,12,14-24)$ and two patients $(13,24)$ underwent MRI examinations. Ultrasound was performed in 12 cases $(11-15,17-21,23)$ but celiac angiography was only used in one case (24). These imagined examinations help surgeons make an accurate diagnosis. In the 15 cases, only two of them $(12,16)$ were malignant and pre-diagnosis included pNET, gastrointestinal stromal tumor (GIST), sarcoma and PGL. Fourteen patients (11-17, 


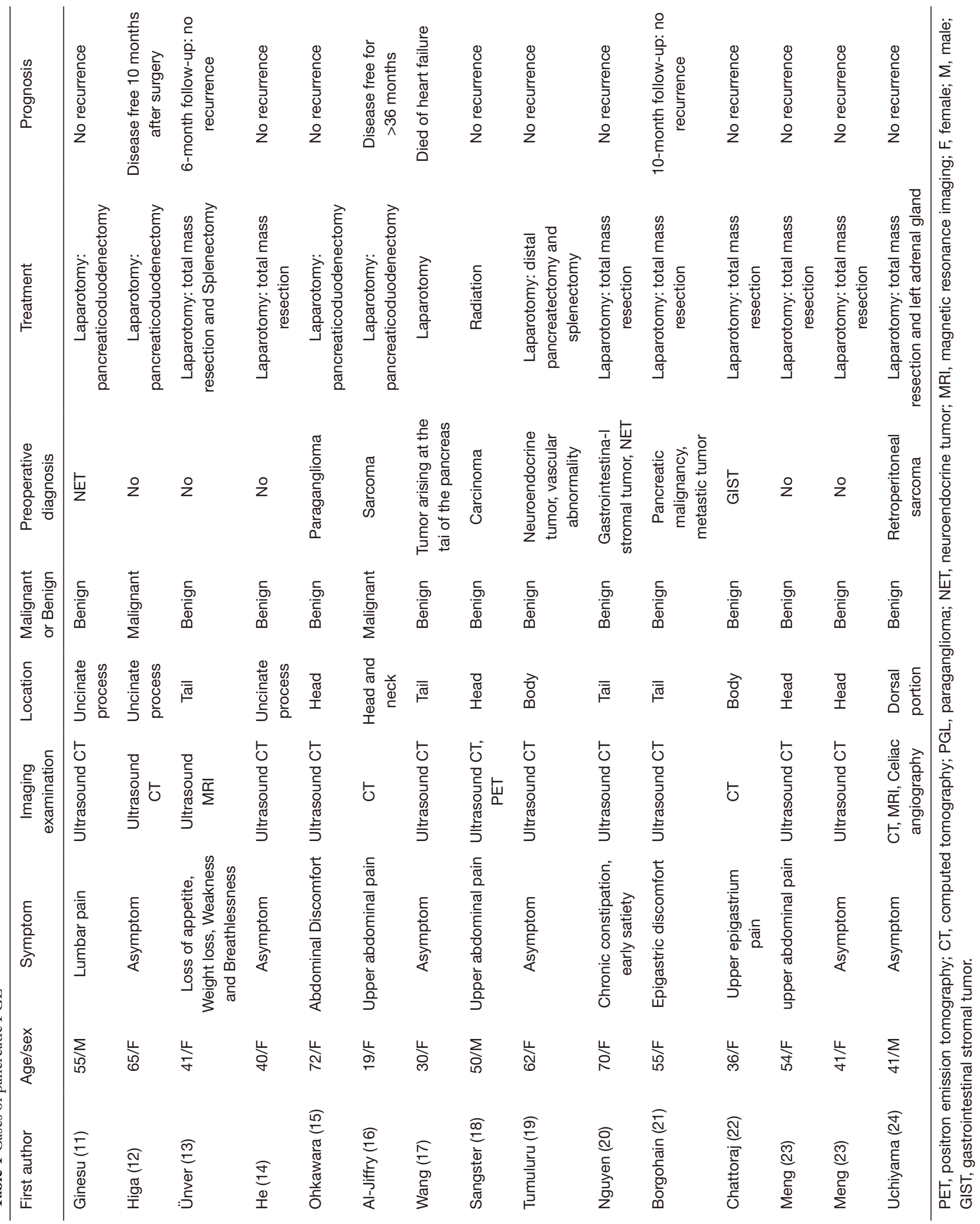


19-24) underwent surgery and one (18) was treated by radiation. Prognosis of the fourteen patients (11-16, 18-24) were good and only one (17) died of heart failure. Open radical surgery like distal pancreatectomy combined with splenectomy, pancreaticoduodenectomy, total mass resection combined with splenectomy or total mass resection combined with the left adrenal gland was performed in seven cases. $(11-13,15,16,19,24)$ The others $(14,17,20-23)$ underwent simple open resection of the mass without excessive operation. In our case, the tumor was simply resected by laparoscopic technique without excessive surgical treatment and laparotomy. And the prognosis for simple excision of the tumor was equally good to radical surgery such as pancreaticoduodenectomy. Additionally, laparoscopic technique alleviates the patient's injuries and shortens the postoperative in-hospital day, and this is a feature of our case.

There are several differences among PGL, Castleman's disease and pNET (Table 2). The occurrence of PGL is usually associated with some genetic mutations: succinate dehydrogenase (SDH) subunits (SDHx), Von HippelLin (VHL), endothelial PAS domain protein 1 (EPAS1)/ hypoxia-inducible factor $2 \alpha$ (HIF2A) and prolyl hydroxylase $1 / 2$ (PHD1/2) (25). The tumor may be associated with hypertension or fluctuation in blood pressure, and internal bleeding and cystic changes are common. Clinical manifestations may be asymptomatic or symptoms of local compression. Blood and urine catecholamine is abnormal in some patients. The enhanced CT scan shows the tumor is significantly enhanced at the early stage. The main treatment is surgical removal and the majority of the patient have good prognosis. Castleman's disease is segmented into un-centric Castleman's disease (UCD) and multicentric Castleman's disease (MCD). Castleman's disease is mainly located in the mediastinum, head, neck, abdomen, retroperitoneum and generalized lymphadenopathy. EB virus (EBV), human herpesvirus (HHV-8), IL-6 and human immunodeficiency virus (HIV) are closely related to the disease. The UCD is more common with no obvious symptoms but $\mathrm{CD}$ often had anemia, fever and night sweats, and serological examination shows that steroids, IL-6, C-reactive protein (CRP), and gamma-globulin were abnormal. Castleman's disease is usually presented as a well-defined solid mass with uniform density, progressive enhancement and continuous enhancement in enhanced CT. UCD should be removed by surgery and usually has good prognosis. However, the CD should be treated by a combination of surgery, radiotherapy and chemotherapy.

As for the pNET, it often has the mutation of $\alpha$-thalassaemia/mental retardation syndrome $\mathrm{X}$-linked (ATRX)/death-domain associated protein (DAXX), ATrich interactive domain-containing protein $1 \mathrm{~A}$ (ARID1A), multiple endocrine neoplasia type 1 (MEN-1), mutY homolog (MUTYH) and mammalian target of rapamycin (mTOR) (26). The tumor is malignant and located in the pancreas. It is classified as non-functional pNET and functional pNET. The non-functional pNET is asymptomatic or has a symptom of local compression, but the functional pNET is presented as carcinoid syndrome, Whipple's triplet syndrome, Zollinger-Ellison syndrome, glucagonoma syndrome and Vemer-Morrison syndrome. The tumor markers for Cg A, Syn, PPP and NSE can be high in the blood (27). In the MRI images, the tumor presents hypointense for T1WI. On T2WI, it mostly shows hyperintense but a few of them are in isointense or hypointense. As for the non-functional pNET in the enhanced CT images, the diameter of the tumor mostly exceeds $5 \mathrm{~cm}$ and the tumor shows heterogeneous enhancement, necrosis and cystic degeneration. Functional pNET often has a smaller diameter $(<2 \mathrm{~cm})$, clear boundary and rich blood supply in the enhanced CT images. Radical resection is the main treatment measure and it can be combined with radiotherapy, chemotherapy and targeted therapy.

\section{Conclusions}

Retroperitoneal PGL in the pancreas is extremely rare and surgeons often have difficulties in distinguishing PGL from Castleman's disease or pNET, so the pathological examination is necessary for an accurate diagnosis. We successfully resected the tumor using the laparoscopic technique. Fifteen cases were listed to show the characters of PGL locating in the pancreas and we listed the differences among PGL, Castleman's disease or pNET. We introduced our experience of treating such a rare tumor hoping that it will help clinicians to correctly diagnose and treat PGL.

\section{Patient perspective}

The patient appreciated our surgery because it relieved the pain and removed her concerns about the tumor. 
Table 2 Differential diagnosing among PGL, Castleman's disease and pancreatic neuroendocrine tumor

\begin{tabular}{|c|c|}
\hline & Paraganglioma \\
\hline Origin & Paraganglia cell \\
\hline Etiology & $\begin{array}{l}\text { Genetic mutations: SDHx, VHL, } \\
\text { EPAS1/HIF2A, PHD1/2 }\end{array}$ \\
\hline Classification & $\begin{array}{l}\text { Parasympathetic paraganglia, } \\
\text { sympathetic paraganglia }\end{array}$ \\
\hline Locations & $\begin{array}{l}\text { Parasympathetic paraganglia: } \\
\text { posterior mediastinum or } \\
\text { retroperitoneum } \\
\text { Sympathetic paraganglia: head and } \\
\text { neck, anterior/middle mediastinum }\end{array}$ \\
\hline Malignant risk & Low \\
\hline Symptoms & $\begin{array}{l}\text { Asymptomatic, fluctuations of blood } \\
\text { pressure, compression }\end{array}$ \\
\hline
\end{tabular}

Laboratory Catecholamine in blood and urine inspection

MRI

CT

Pathology

Treating

\author{
$\mathrm{T} 1$ : isointense \\ T2: hyperintense, hypointense or \\ mixed intensity
}

Enhanced CT:

Significantly enhanced at early stage
Tumor cells: polygonal, arranged in
clusters or nests. CK (pan) (-), Ki-67 $(+,<1 \%$,$) , Melan A (-), Cg A (+), Syn$ (+), S-100(+)

Castleman's disease
Lymph node
EBV, HHV-8, IL-6, HIV
UCD, MCD
Mediastinum, head and neck,
abdomen, retroperitoneum,
generalized lymphadenopathy
UCD: low
MCD: high
UCD: a symptom
MCD: anemia, fever, night sweats

Steroids, IL-6, CRP and gammaglobulin in blood

T1: well-defined homogeneous isointense-to-muscle masse T2: intermediate contrast enhancement and intermediatehyperintense

Enhanced CT:

Uniform density, progressive enhancement and continuous enhancement
Pancreatic neuroendocrine tumor

Multipotent stem cells

Genetic mutations: ATRX/DAXX, ARID1A, MEN-1, MUTYH, mTOR

Non-functional pNET, functional pNET

Pancreas

Malignant tumor

Non-functional pNET: a symptom or symptoms of local compression Functional pNET: carcinoid syndrome, Whipple's triplet syndrome, Zollinger-Ellison syndrome, glucagonoma syndrome, Vemer-Morrison syndrome

Cg A, Syn, PPP, NSE

T1: hypointense

T2: most was in hyperintense, a few was in isointense or hypointense

\section{Enhanced CT:}

Non-functional pNET: larger tumors may exceed $5 \mathrm{~cm}$ in diameter, heterogeneous enhancement (necrosis, cystic degeneration) Functional pNET: Smaller diameter $(<2 \mathrm{~cm})$, a well-demarcated solid mass and rich blood supply

Cg A (+), Syn (+), E-cad (+), CD 56 (+), NSE (+)
CD20+ T cell, CD79a $+\mathrm{T}$ cell, CD 45RO+ T cell and CD45RO+ B cell, CD21+ follicular dendritic cell, CD3(+), CD5(+), CD43(+), Bcl-2(+), $\kappa$ chain $(+), \lambda$ chain $(+)$

Surgery, radiotherapy, chemotherapy
Surgery, radiotherapy, chemotherapy, targeted therapy

SDHx, succinate dehydrogenase subunits; VHL, Von Hippel-Lin; EPAS1, endothelial PAS domain protein 1; HIF2A, hypoxia-inducible factor $2 \alpha$; PHD1/2, prolyl hydroxylase 1/2; CT, computed tomography; MRI, magnetic resonance imaging; Pnet, pancreatic neuroendocrine tumor; CK (pan), cytokeratin pan; Cg A, chromogranin A; Syn, synaptophysin; EBV, epstein-barr virus; HHV-8, human herpes virus-8; IL6, interleukin-6; HIV, human immunodeficiency virus; CRP, c-reactive protein; PPP, pancreatic polypeptide; NSE, neuron specific enolase; ATRX, $\alpha$-thalassaemia/mental retardation syndrome X-linked; DAXX, death-domain associated protein; ARID1A, AT-rich interactive domaincontaining protein 1A; MEN-1, multiple endocrine neoplasia type 1; MUTYH, mutY homolog; mTOR, mammalian target of rapamycin; UCD, un-centric Castleman's disease; MCD, multicentric Castleman's disease; E-cad, e-cadherin. 


\section{Acknowledgments}

Funding: Zhejiang Provincial Natural Science Foundation of China (No. LQ16H030002) and Zhejiang Medical and Health Science and Technology Project (No. 2016KYB086).

\section{Footnote}

Reporting Checklist: The authors have completed the CARE reporting checklist. Available at http://dx.doi.org/10.21037/ gs-20-758

Peer Review File: Available at http://dx.doi.org/10.21037/ gs20-758

Conflicts of Interest: All authors have completed the ICMJE uniform disclosure form (available at http://dx.doi. org/10.21037/ gs-20-758). The authors have no conflicts of interest to declare.

Ethical Statement: The authors are accountable for all aspects of the work in ensuring that questions related to the accuracy or integrity of any part of the work are appropriately investigated and resolved. All procedures performed in studies involving human participants were in accordance with the ethical standards of the institutional and/or national research committee(s) and with the Helsinki Declaration (as revised in 2013). Written informed consent was obtained from the patient. The study was approved by the ethics committee of The First Affiliated Hospital, School of Medicine, Zhejiang University (No. IIT20200277A).

Open Access Statement: This is an Open Access article distributed in accordance with the Creative Commons Attribution-NonCommercial-NoDerivs 4.0 International License (CC BY-NC-ND 4.0), which permits the noncommercial replication and distribution of the article with the strict proviso that no changes or edits are made and the original work is properly cited (including links to both the formal publication through the relevant DOI and the license). See: https://creativecommons.org/licenses/by-nc-nd/4.0/.

\section{References}

1. Corssmit EP, Romijn JA. Clinical management of paragangliomas. Eur J Endocrinol 2014;171:R231-43.

2. Chen H, Sippel RS, O'Dorisio MS, et al. The
North American Neuroendocrine Tumor Society consensus guideline for the diagnosis and management of neuroendocrine tumors: pheochromocytoma, paraganglioma, and medullary thyroid cancer. Pancreas 2010;39:775-83.

3. Lin B, Yang H, Yang H, et al. Bilateral malignant paragangliomas in a patient: a rare case report. World Neurosurg 2019. [Epub ahead of print]. doi: 10.1016/ j.wneu.2018.12.131.

4. Purnell S, Sidana A, Maruf M, et al. Genitourinary paraganglioma: Demographic, pathologic, and clinical characteristics in the surveillance, epidemiology, and end results database (2000-2012). Urol Oncol 2017;35:457.e9457.e14.

5. Yang $\mathrm{Y}$, Wang G, Lu H, et al. Haemorrhagic retroperitoneal paraganglioma initially manifesting as acute abdomen: a rare case report and literature review. BMC Surg 2020;20:304.

6. Wang Z, Yang J. Primary retroperitoneal paraganglioma mimicking a ureteral tumor: a case report and literature review. Postgrad Med 2020;132:657-61.

7. Taïeb D, Hicks RJ, Hindié E, et al. European Association of Nuclear Medicine Practice Guideline/ Society of Nuclear Medicine and Molecular Imaging Procedure Standard 2019 for radionuclide imaging of phaeochromocytoma and paraganglioma. Eur J Nucl Med Mol Imaging 2019;46:2112-37.

8. Layfield LJ, Gopez EV. Percutaneous image-guided fineneedle aspiration of peritoneal lesions. Diagn Cytopathol 2003;28:6-12.

9. Walz MK, Peitgen K, Neumann HP, et al. Endoscopic treatment of solitary, bilateral, multiple, and recurrent pheochromocytomas and paragangliomas. World J Surg 2002;26:1005-12.

10. Cunningham SC, Suh HS, Winter JM, et al. Retroperitoneal paraganglioma: single-institution experience and review of the literature. J Gastrointest Surg 2006;10:1156-63.

11. Ginesu GC, Barmina M, Paliogiannis P, et al. Nonfunctional paraganglioma of the head of the pancreas: A rare case report. Int J Surg Case Rep 2016;28:81-4.

12. Higa B, Kapur U. Malignant paraganglioma of the pancreas. Pathology 2012;44:53-5.

13. Ünver M, Öztürk Ş, Erol V, et al. Retroperitoneal paraganglioma presenting with pancytopenia: A rare case with rare manifestation. Int J Surg Case Rep 2015;14:77-9.

14. He J, Zhao F, Li H, et al. Pancreatic paraganglioma: A case report of CT manifestations and literature review. Quant 
Imaging Med Surg 2011;1:41-3.

15. Ohkawara T, Naruse H, Takeda H, et al. Primary paraganglioma of the head of pancreas: contribution of combinatorial image analyses to the diagnosis of disease. Intern Med 2005;44:1195-6.

16. Al-Jiffry BO, Alnemary Y, Khayat SH, et al. Malignant extra-adrenal pancreatic paraganglioma: case report and literature review. BMC Cancer 2013;13:486.

17. Wang ZL, Fu L, Zhang Y, et al. An asymptomatic pheochromocytoma originating from the tail of the pancreas. Pancreas 2012;41:165-7.

18. Sangster G, Do D, Previgliano C, et al. Primary retroperitoneal paraganglioma simulating a pancreatic mass: a case report and review of the literature. HPB Surg 2010;2010:645728.

19. Tumuluru S, Mellnick V, Doyle M, et al. Pancreatic Paraganglioma: A Case Report. Case Rep Pancreat Cancer 2016;2:79-83.

20. Nguyen E, Nakasaki M, Lee TK, et al. Diagnosis of paraganglioma as a pancreatic mass: A case report. Diagn Cytopathol 2018;46:804-6.

21. Borgohain M, Gogoi G, Das D, et al. Pancreatic

Cite this article as: Wang W, Qin Y, Zhang H, Chen K, Liu Z, Zheng S. A rare case of retroperitoneal paraganglioma located in the neck of the pancreas: a case report and literature review. Gland Surg 2021;10(4):1523-1531. doi: 10.21037/gs-20-758 paraganglioma: An extremely rare entity and crucial role of immunohistochemistry for diagnosis. Indian J Endocrinol Metab 2013;17:917-9.

22. Chattoraj AK, Rao UM, Sarkar N, et al. Non-functional retroperitoneal paraganglioma: A case report. J Family Med Prim Care 2019;8:1497-9.

23. Meng L, Wang J, Fang SH. Primary pancreatic paraganglioma: a report of two cases and literature review. World J Gastroenterol 2015;21:1036-9.

24. Uchiyama S, Ikenaga N, Haruyama Y, et al. Asymptomatic extra-adrenal paraganglioma masquerading as retroperitoneal sarcoma. Clin J Gastroenterol 2010;3:13-7.

25. Pillai S, Gopalan V, Lo CY, et al. Silent genetic alterations identified by targeted next-generation sequencing in pheochromocytoma/paraganglioma: A clinicopathological correlations. Exp Mol Pathol 2017;102:41-6.

26. Han X, Lou W. Advances in Research on Genotyping and the Molecular Mechanism of Pancreatic Neuroendocrine Neoplasias. Med J PUMCH 2020;11:377-82.

27. Li X, Cui Y. The clinical characteristics and comprehensive treatment progress of pancreatic neuroendocrine neoplasm. Abdominal Surg 2020;33:391-5. 